\title{
An application of composite complex sinusoidal modeling to the estimation of direction and spectra of an incident plane wave
}

\author{
Masato Abe and Ken'iti Kido \\ Research Center for Applied Information Sciences, \\ Tohoku University, \\ 2-1-1, Katahira, Sendai, 980 Japan
}

(Received 5 January 1982)

\begin{abstract}
The present paper proposes composite complex sinusoidal modeling (CCSM) for the estimation of direction and spectrum of an incident sound using a linear array of microphones. Output signals of the microphones in the array are stored in a computer and separated by discrete Fourier transform (DFT) into different frequency components, for all of which spatial sequences are generated. If only one plane wave is present, a spatial complex sinusoidal wave is formed by the sequence, whereas in the case of a plural number of plane waves, the sequence is provided by the sum of the same number of spatial complex sinusoidal waves as that of the plane waves. Direction and spectrum of an incident plane wave are estimated by evaluating amplitude and a rate of phase change (PCR: phase change rate) of the corresponding sinusoidal wave. The directivity pattern obtained by CCSM has no side lobe and gives only a single line which indicates direction and power of the incident plane wave even if the length of the array is of the same order as the wave length of the incident plane wave. In addition, effectiveness of CCSM is confirmed in computer simulation.
\end{abstract}

PACS number: 43. 50. Yw, 43. 60. Gk

\section{INTRODUCTION}

The composite sinusoidal modeling was first proposed for real data to be used for speech analysis and synthesis, ${ }^{1)}$ and then developed for complex data to be used for array signal processing. ${ }^{2)}$

The output of a microphone in a linear array is A/D converted and stored in a computer for a certain length of time, which should sufficiently be longer than the duration in which an incident sound propagates from one end of the array to the other end. A stored sequence is cut out by a proper time window and separated into different frequency components by Fourier transform. After selecting the same frequency components from spectra of the outputs of all microphones, a new sequence is generated for every frequency component.
With more than one plane wave coming in, the sequence is represented by the sum of the same number of spatial complex sinusoidal waves as that of the plane waves. Direction and power of the frequency component of a plane wave are estimated by evaluating amplitude and phase change rate (PCR) of the corresponding complex sinusoidal wave. Thus, any method for estimating the spectrum of a signal could be applied to the estimation of direction and spectrum of an incident plane wave. Discrete Fourier transform (DFT) is generally used for the estimation of amplitude and PCR of a discrete complex sinusoidal wave. However, a sharp directivity pattern is not obtained by Fourier. transform unless the length of the array is much longer than the wave length of an incident sound. Furthermore, a directivity pattern obtained by DFT 
yields many side lobes. Maximum entropy method (MEM) has been proposed to realize sharper directivity and to eliminate side lobes in the directivity pattern. However, this method is not good enough because the main lobe of the directivity pattern is still wide. Composite complex sinusoidal modeling (CCSM) proposed in this paper is based on the assumption that the spatial sequence of a frequency component is composed of a number of complex sinusoidal waves. A directivity pattern obtained by CCSM has no side lobe, but shows rather a single line. A single line indicating direction and power of an incident plane wave is obtained even if the length of the array is of the same order as the wave length of the plane wave.

In the present paper, the methods for the estimation of direction and spectrum of an incident plane wave are described, whereas effectiveness of CCSM is also confirmed in computer simulation.

\section{PRINCIPLE}

It is assumed that a plane wave comes from the direction $\theta$ to the uniformly spaced array composed of $N$ microphones named $\mathbf{M}_{0}, \mathbf{M}_{1}, \cdots, \mathbf{M}_{N-1}$ as shown in Fig. 1. The output of the microphone $\mathbf{M}_{0}$ being $x_{0}(t)$, the output of the microphone $\mathbf{M}_{n}$ is given as

$$
x_{n}(t)=x_{0}(t-n d \cos \theta / c),
$$

where $d$ denotes the distance between two adjacent microphones and $c$ the sound velocity. The distance $d$ should be less than a half of the wave length of an incident sound in order to avoid the effect of spatial aliasing which may produce a ghost of sound source in the direction where there is no sound source.

In the plane wave composed of a single frequency component $f_{0}$, the phase difference $\psi$ between two adjacent microphones is expressed using the PCR $\phi$ as

$$
\begin{aligned}
\psi & =\phi d \\
& =-2 \pi f_{0} d \cos \theta / c,
\end{aligned}
$$

where

$$
\phi=-2 \pi f_{0} \cos \theta / c .
$$

The phase difference between $x_{0}(t)$ and $x_{n}(t)$ at frequency $f_{0}$ is represented in complex number as follows:

$$
\begin{aligned}
\Phi_{n} & =\exp (j \phi n d) \\
& =\exp \left(-j 2 \pi f_{0} n d \cos \theta / c\right) .
\end{aligned}
$$

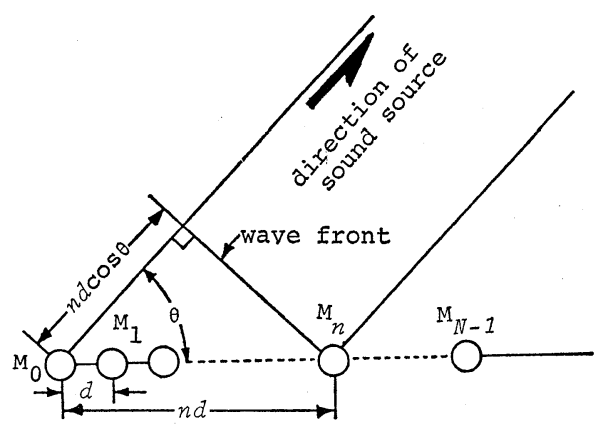

Fig. 1 Relation between a microphone array and an incident plane wave.

Thus, as shown in Fig. 2, a complex sinusoidal wave is formed by a spatial sequence obtained by arranging the complex phase differences $\Phi_{n}$ according to the order of microphone number $n$. It is observed from Figs. 2(a) and 2(b) that the spatial period of the sinusoidal wave on the array is shorter in Fig. 2(a), where the incident angle $\theta=30^{\circ}$, than in Fig. 2(b), where $\theta=70^{\circ}$. Figures 2(c) and 2(d) show the relation of the sinusoidal waves between the case $\theta=90^{\circ}-30^{\circ}$ and the case $\theta=90^{\circ}+30^{\circ}$; the phase is reversed when the incident angle $\theta$ exceeds $90^{\circ}$. It is clear that the direction $\theta$ of the incident plane wave can be estimated by evaluating the $\operatorname{PCR} \phi$ when $f_{0}$ and $c$ are known.

When a number of incident plane waves come from different directions, a spatial sequence is given by the sum of the same number of complex sinusoidal waves as that of the incident plane waves, the direction of which is estimated by evaluating the PCR $\phi$ of the corresponding complex sinusoidal wave.

Direction of an incident plane wave can be estimated correctly only when the frequency is fixed. Therefore, when the frequency of an incident plane wave is changing, the direction should be estimated after fixing the frequency using a method for spectral analysis such as discrete Fourier transform.

The outputs of the microphones are band-limited using an appropriate analog low pass filters and $\mathrm{A} / \mathrm{D}$ converted at a sampling rate of $f_{s}$. Let the $m$-th sample of an output of the $n$-th microphone be $x_{n}^{\prime}(m)$, then

$$
\begin{aligned}
x_{n}^{\prime}(m) & =x_{n}\left(m / f_{s}\right) \\
& =x_{0}\left(m / f_{s}-n d \cos \theta / c\right) .
\end{aligned}
$$




\section{ABE and K. KIDO: APPLICATION OF CCSM TO ARRAY SIGNAL PROCESSING}

The sequence $x_{n}^{\prime}(m)$ is cut out by multiplying Hanning window $h(m)$ with the length $M$ and is separated into different frequency components by discrete Fourier transform (DFT). The observed time corresponding to the length $M$ of Hanning window should sufficiently be long so that the outputs of the microphones are highly correlated with one another; the duration of time window should be longer than the duration, in which an incident sound propagates from one end of the array to the other end. Thus, the $k$-th frequency component $X_{n}(k)$ is expressed as

$$
X_{n}(k)=\frac{1}{M} \sum_{m=0}^{M-1} h(m) x_{n}^{\prime}(m) \exp (-j 2 \pi m k / M),
$$

where

$$
h(m)= \begin{cases}1-\cos (2 \pi m k / M), & 0 \leqq m \leqq M-1 \\ 0, & \text { otherwise } .\end{cases}
$$

Since the observed time $T$ corresponding to the length $M$ is sufficiently long, the spectrum of each sequence $h(m) x_{n}^{\prime}(m)$ differs from one another only in phase. Hence, Eq. (5) is expressed approximately as

$$
\begin{aligned}
X_{n}(k) & \doteqdot X_{0}(k) \Phi_{n} \\
& =X_{0}(k) \exp \left(-j 2 \pi \frac{k}{T} n d \cos \theta / c\right),
\end{aligned}
$$

where

$$
\begin{aligned}
T & =M / f_{s}, \\
\Phi_{n} & =\exp (j \phi n d) \\
& =\exp \left(-j 2 \pi \frac{k}{T} n d \cos \theta / c\right)
\end{aligned}
$$

and $k / T$ corresponds to the frequency $f_{0}$ by which the direction of an incident plane wave is estimated.

Here, the following equation is obtained using the relation $-1 \leqq \cos \theta \leqq+1$.

$$
-\frac{2 \pi k}{T c} \leqq \phi \leqq+\frac{2 \pi k}{T c} .
$$

It is clear from Eq. ( $\left(7^{\prime}\right)$ that a sequence expressing a spatial complex sinusoidal wave is obtained by arranging the $k$-th frequency components according to the order of microphone number $n$. Therefore, the power spectrum $\left|X_{0}(k)\right|^{2}$ and the direction $\theta$ of the incident plane wave can be estimated by evaluating the amplitude $\left|X_{0}(k)\right|$ and the PCR $\phi$ of the complex sinusoidal wave.
When $P$ plane waves come in from different directions as shown in Fig. 3, the spectrum of an output of microphone $\mathbf{M}_{n}$ is expressed as

$$
\begin{aligned}
X_{n}(k) & =\sum_{p=1}^{P} A_{p}(k) \exp \left(j \phi_{p} n d\right) \\
& =\sum_{p=1}^{P} A_{p}(k) \exp \left(-j 2 \pi \frac{k}{T} n d \cos \theta_{p} / c\right),
\end{aligned}
$$

where $A_{p}(k)$ represents the spectrum of an output of microphone $\mathrm{M}_{0}$ for the $p$-th plane wave with the incident angle $\theta_{p}$.

It is clear from Eq. (11) that the spatial sequence $X_{n}(k), n=0,1, \cdots, N-1$, is composed of $P$ complex sinusoidal waves with the amplitude $A_{p}(k)$ and the PCR $\phi_{p}$. Therefore, the power spectrum $\left|A_{p}(k)\right|^{2}$ and the direction $\theta_{p}$ of an incident plane wave, where $p=1,2, \cdots, P$, can be estimated by evaluating the amplitude $\left|A_{p}(k)\right|$ and the PCR $\phi_{p}$. The relation between angular frequency $\omega$ and time $t$ is the same as that between the PCR $\phi$ and the distance $n d$ between microphones. Thus, an arbitrary method for estimating the spectrum of a signal can be applied to the estimation of power and direction of an incident plane wave.

The direction and the power spectra of an incident plane wave can be estimated by executing the above processing for every frequency components.

\subsection{Estimation by DFT}

DFT is generally used for estimating the amplitude of every frequency component of a signal. Therefore, DFT can be applied to the estimation of amplitude of a spatial sequence at every discrete component of PCR. In order to estimate the power at every component, the sequence $X_{n}(k)$, where $n=0,1, \cdots, N-1$, is Fourier transformed as

$$
\begin{aligned}
Y(q)= & \frac{1}{N} \sum_{n=0}^{N-1} X_{n}(k) \exp (-j 2 \pi q n / N) \\
= & \frac{1}{N} \sum_{n=0}^{N-1} \sum_{p=1}^{P} A_{p}(k) \exp \left(-j 2 \pi \frac{k}{T} n d \cos \theta_{p} / c\right) \\
& \cdot \exp (-j 2 \pi q n / N) \\
= & \frac{1}{N} \sum_{n=0}^{N-1} \sum_{p=1}^{P} A_{p}(k) \exp \{-j 2 \pi \\
& \left.\cdot\left(\frac{k}{T} \cos \theta_{p} / c+q / L\right) n d\right\},
\end{aligned}
$$

where $L$ is the length of the array and is given as

$$
L=N d \text {. }
$$


REAL

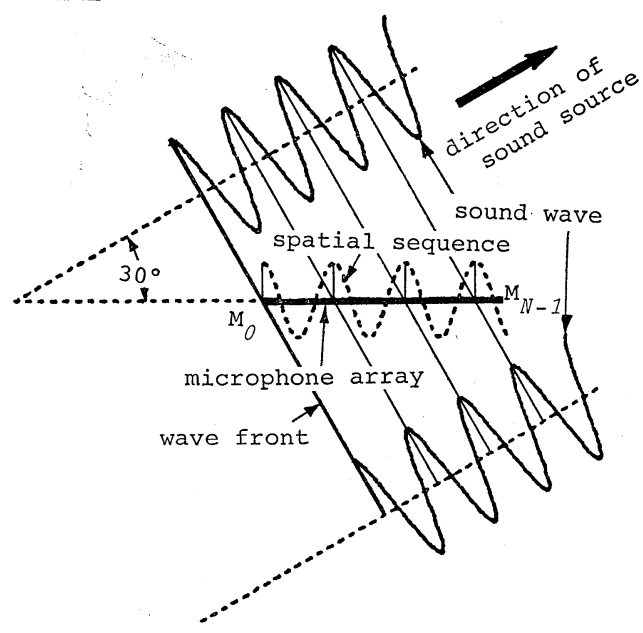

IMAG.

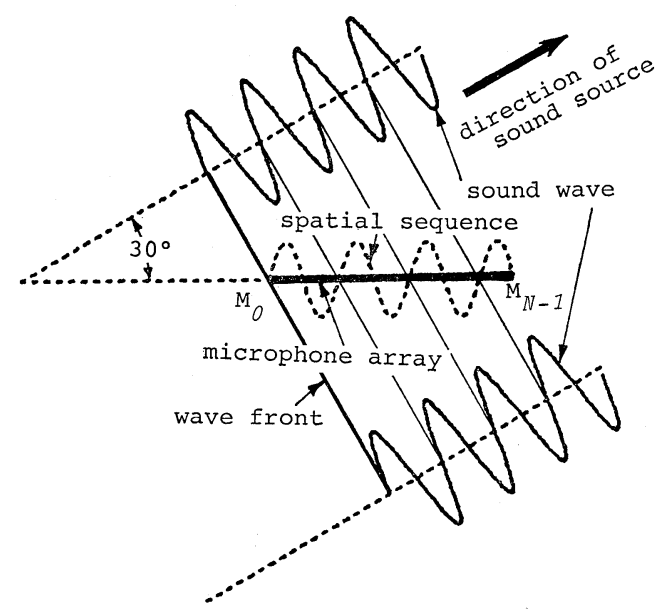

Fig. 2 (a)

REAL

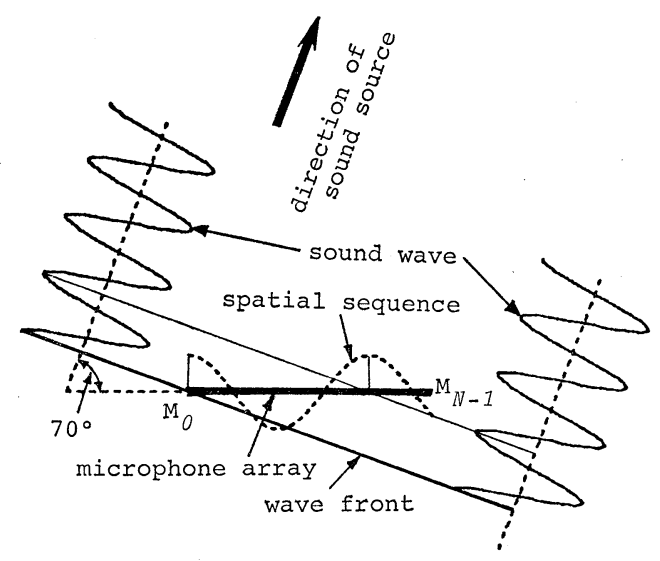

IMAG.

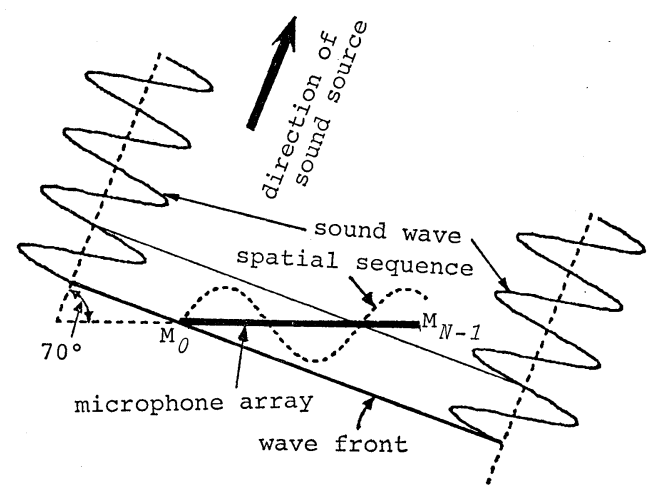

Fig. 2(b)

It is clear from Eq. (12) that $Y(q)$ gives peaks at the PCR $\phi_{p}$, where

$$
\begin{gathered}
\phi_{p}=2 \pi q_{p} / L=-2 \pi \frac{k}{T} \cos \theta_{p} / c, \\
p=1,2, \cdots, P
\end{gathered}
$$

and $Y\left(q_{p}\right)$ is the spectrum estimate of the $p$-th plane wave because the following equation is obtained when the array length $L$ is much greater than the wave length of an incident plane wave and $d$ is much less than $L$.

$$
Y\left(q_{p}\right)=A_{p}(k)+\sum_{\substack{i=1 \\ i \neq p}}^{P} A_{i}(k) \cdot \frac{1}{N}
$$

$$
\begin{aligned}
& \frac{1-\exp \left\{-j 2 \pi\left(\frac{k}{T} \cos \theta_{i} / c+q_{p} / L\right) L\right\}}{1-\exp \left\{-j 2 \pi\left(\frac{k}{T} \cos \theta_{i} / c+q_{p} / L\right) d\right\}} \\
\fallingdotseq & A_{p}(k)+\sum_{\substack{i=1 \\
i \neq p}}^{P} A_{i}(k) \\
& \cdot \frac{1-\exp \left\{-j 2 \pi\left(\frac{k}{T} \cos \theta_{i} / c+q_{p} / L\right) L\right\}}{j 2 \pi\left(\frac{k}{T} \cos \theta_{i} / c+q_{p} / L\right) N d} \\
\doteqdot & A_{p}(k) .
\end{aligned}
$$



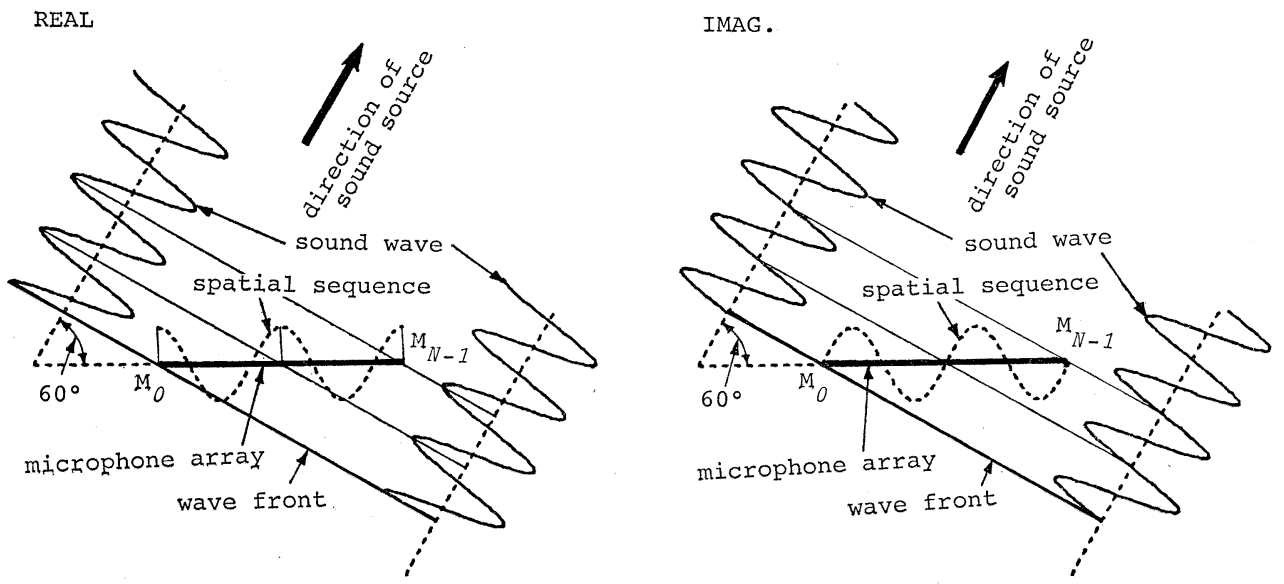

Fig. $2(c)$
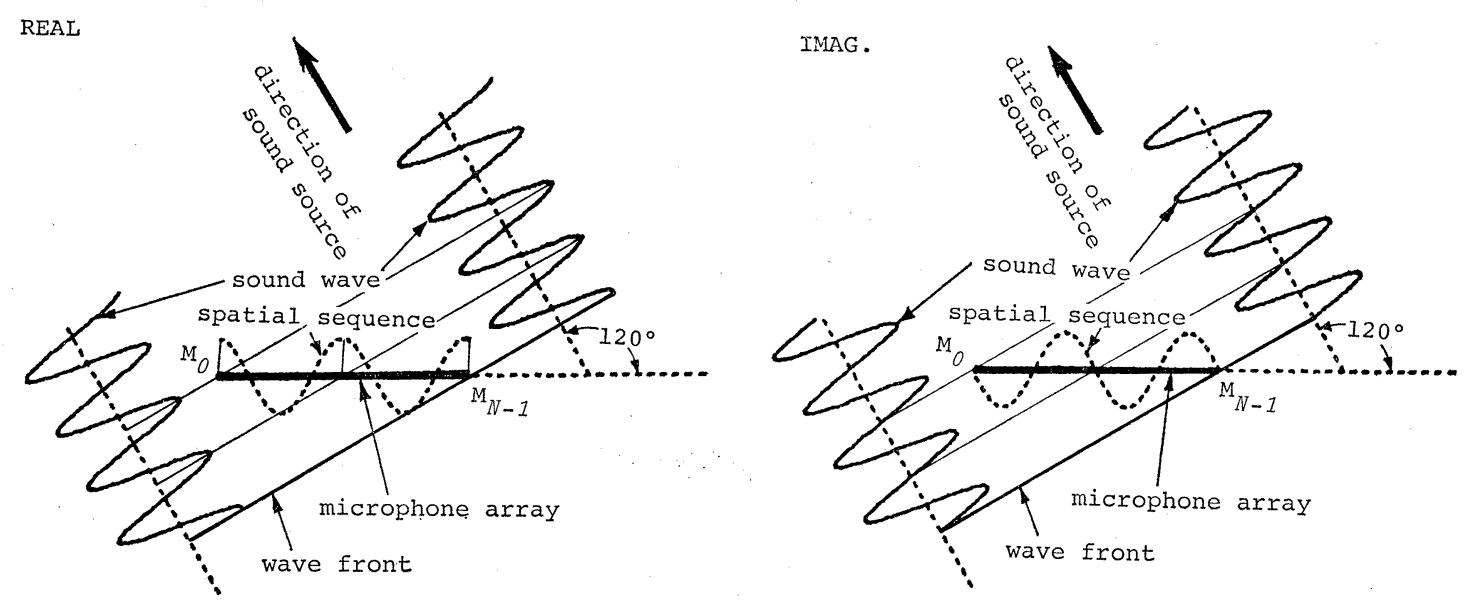

Fig. 2 (d)

Fig. 2 Relation between the incident angle of a plane wave and the spatial sequences on a microphone array with the length being 4 times as long as the wave length of the sound. (a) $\theta=30^{\circ}$, (b) $\theta=70^{\circ}$, (c) $\theta=60^{\circ}$, (d) $\theta=120^{\circ}$.

The direction $\theta_{p}$ and the power spectrum $\left|A_{p}(k)\right|^{2}$ of every incident plane wave is estimated using Eqs. (14) and (15), respectively:

\subsection{Estimation by MEM}

Maximum entropy method (MEM) was first proposed by Burg for achieving high-resolution spectral estimates, ${ }^{3)}$ and was later proved to be identical to linear predictive deconvolution filtering and all pole modeling of the spectrum. Direction and spectrum of an incident plane wave can be estimated using MEM as well as DFT because MEM is also one of the methods for computing spectra of a signal. Therefore, MEM was applied to the array signal processing for the estimation of direction and spectrum of an incident plane wave., ${ }^{4,5)}$ Application of MEM to the array signal processing means that the signal composed of complex sinusoidal waves is approximately analyzed by all pole modeling. That is, the decaying sinusoidal waves are approximated by the sinusoidal waves in MEM analysis.

The true number $P$ of incident plane waves is unknown. Therefore, assuming first that the num- 


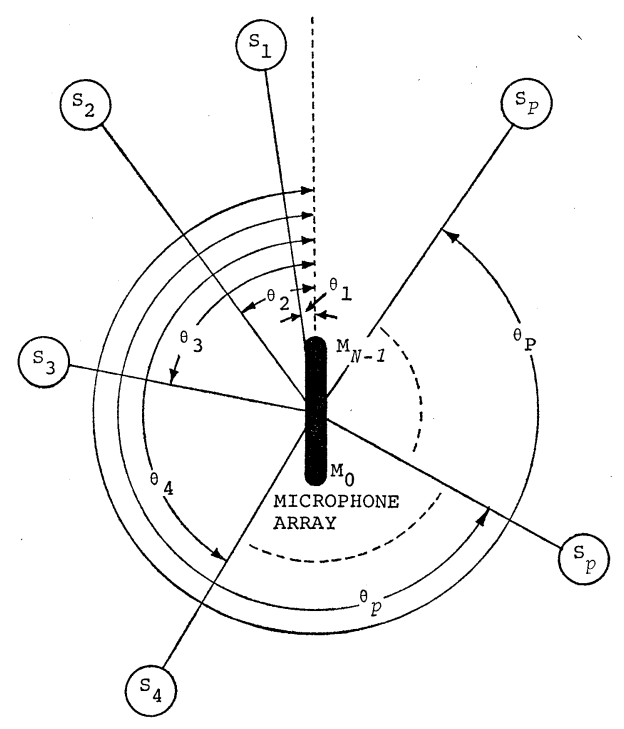

Fig. 3 Relation between a microphone array and $P$ sound sources.

ber $I$ of incident plane waves is one, direction, spectrum and the prediction error are computed. The same operations are repeated incrementing the value $I$ from 1 to a reasonable number. The estimated number of incident plane waves is set to the number $I$ at which the prediction error reaches the smallest value. The estimated direction and the power of each plane wave are those computed for the number $I$.

When it is assumed that there are $I$ incident plane waves, the power spectrum estimate obtained by MEM is a function of PCR $\phi_{q}$ as follows:

$$
Y(q)=\frac{d}{2 L-d} \cdot \frac{P_{I}}{1+\sum_{i=1}^{I} \gamma_{i} \exp \left(-j \phi_{q} i d\right)},
$$

where $1, \gamma_{1}, \cdots, \gamma_{I}$ are the coefficients of the prediction error filter with the length $I+1$, and $P_{I}$ the prediction error. The direction $\theta_{q}$ of an incident plane wave is estimated from Eq. (14) using the PCR $\phi_{q}$ at which Eq. (16) shows a peak. The power spectrum of an incident plane wave is estimated from Eq. (16) also using the PCR $\phi_{q}$; direction and spectrum of an incident plane wave is estimated by determining the length $I+1$ and the coefficients $\gamma_{i}$ of the prediction error filter, and the prediction error $P_{I}$, where $i=1,2, \cdots, I$.

When the length $I+1$ of the prediction error filter is known, the coefficients $\gamma_{i}, i=1,2, \cdots, I$, of the filter are calculated as follows.

Generally, $Y(q)$ is expressed as follows:

$$
Y(q)=\frac{1}{2 N-1} \sum_{k=1-N}^{N-1} c(k) \exp \left(-j \phi_{q} k d\right),
$$

where $c(k)$, where $k=-N+1,-N+2, \cdots,-1,0$, $1, \cdots, N-1$, is the auto-correlation sequence. Comparing Eq. (16) with Eq. (17), the following equations are obtained:

$$
\begin{aligned}
& c(0)+\gamma_{1} c(-1)+\gamma_{2} c(-2)+\cdots+\gamma_{I} c(-I)=P_{I} \\
& c(1)+\gamma_{1} c(0)+\gamma_{2} c(-1)+\cdots+\gamma_{I} c(1-I)=0
\end{aligned}
$$

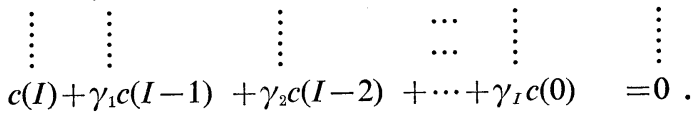

Levinson's algorithm is one of the method for solving Eqs. (18) using recursive relation; the coefficients $\gamma_{I, i}$, where $i=1,2, \cdots, I$, of prediction error filter with the length of $I+1$ are calculated from the coefficients $\gamma_{I-1, i}$ of the filter with the length of $I$. The detail of the determination of the coefficients for real data is developed by Markel and Gray ${ }^{6)}$ The results of the algorithm for complex data are expressed as follows:

$$
\left.\begin{array}{c}
\gamma_{I, I}=\frac{-2 \sum_{i=0}^{N-I-1}\left(b_{I, i}^{*} b_{I, i}^{\prime}\right)}{\sum_{i=0}^{N-I-1}\left\{\left|b_{I, i}\right|^{2}+\left|b_{I, i}^{\prime}\right|^{2}\right\}}, \\
b_{I, i}=b_{I-1, i}+\gamma_{I-1, I-1}^{*} \cdot b_{I-1, i}^{\prime} \\
b_{I, i}^{\prime}=b_{I-1, I+1}^{\prime}+\gamma_{I-1, I-1} \cdot b_{I-1, i+1} \\
b_{0, i}=X_{i}(k) \\
b_{0, i}^{\prime}=X_{i}(k) \\
b_{1, i}=X_{i}(k) \\
b_{1, i}^{\prime}=X_{i+1}(k) \\
{\left[\begin{array}{c}
\gamma_{I, 1} \\
\gamma_{I, 2} \\
\vdots \\
\vdots \\
\gamma_{I, I-1}
\end{array}\right]=\left[\begin{array}{l}
\gamma_{I-1,1} \\
\gamma_{I-1,2} \\
\vdots \\
\gamma_{I-1, I-1}
\end{array}\right]+\gamma_{I, I} \cdot\left[\begin{array}{c}
\gamma_{I-1, I-1}^{*} \\
\gamma_{I-1, I-2}^{*} \\
\vdots \\
\gamma_{I-1,1}^{*}
\end{array}\right],} \\
P_{I}=P_{I-1} \cdot\left(1-\left|\gamma_{I, I}\right|^{2}\right) \\
P_{0}=\frac{1}{N} \sum_{i=0}^{N-1}\left|X_{i}(k)\right|^{2}
\end{array}\right\} \cdot
$$

The remaining problem is to determine the number $I$ of incident plane waves; the length $I+1$ 


\section{ABE and K. KIDO: APPLICATION OF CCSM TO ARRAY SIGNAL PROCESSING}

of the prediction error filter. Skinner ${ }^{5)}$ introduced final prediction error (FPE) as expressed as follows:

$$
\begin{aligned}
F P E_{I}= & \frac{N+(I+1)}{N-(I+1)} \sum_{i=I}^{N-1} \mid X_{i}(k)+\gamma_{I, 1} X_{i-1}(k) \\
& +\cdots+\left.\gamma_{I, I} X_{i-I}(k)\right|^{2} .
\end{aligned}
$$

In this paper, the length $I+1$ of the prediction error filter is set to the number where FPE falls the smallest value.

In the estimation using all pole modeling such as MEM, frequency and the magnitude of a peak in transfer function stand for PCR and amplitude of a complex sinusoidal wave, respectively. The incident angle of a plane wave is directly computed by PCR using the wave length and the intervals between microphones, and the amplitude of the incident plane wave is in proportion to the magnitude of the peak. That is, the complex sinusoidal waves on the array are approximated by the sum of decaying sinusoidal waves, and a sinusoidal wave with a small power is approximated by a rapidly decaying sinusoidal wave which corresponds to an impulse response of transfer function with a dull peak. Therefore, an incident plane wave with a small power is expressed by a dull peak, and high resolution in the incident angle can not be obtained. The estimated number $I$ of incident plane waves can not always be equal to the true number $P$ since the composite complex sinusoidal waves are approximated by the sum of decaying sinusoidal waves, and the spectrum can not be always estimated correctly since the spectrum is expressed by all pole modeling.

\subsection{Estimation by CCSM}

Proposed here is a new algorithm for the estimation of the components of complex sinusoidal waves. The analysis by CCSM is based on the assumption that the sequence to be estimated is composed of several complex sinusoidal waves, whereas in MEM analysis it is assumed that a sequence is composed of several decaying complex sinusoidal waves, and the power of each sinusoidal wave is estimated approximately by all pole modeling. Therefore, CCSM is more adequate than MEM for the application to the array signal processing since the spatial sequence made of array signal outputs is represented by the sum of non-decaying complex sinusoidal waves.

The true number $P$ of incident plane waves is unknown. Therefore, directions of incident plane waves are computed, as well as in MEM analysis, incrementing the number $I$ from 1 to a reasonable number, where $I$ is the assumed number of incident plane waves. In the process of computation, the assumed number $I$ of incident plane waves is reduced to $I^{\prime}$ discarding the spatial sinusoidal waves on the array whose spatial period is shorter than the length of the sound at the frequency in question. Here, the assumed number $I$ of incident plane waves is reduced to $I^{\prime}$. Using the number $I^{\prime}$, power of each incident plane wave and the prediction error are computed. The estimated number of incident plane waves is set to the number $I^{\prime}$ at which the prediction error reaches the smallest value. The estimated direction and power are those computed using the number $I^{\prime}$.

Here, it is assumed that there are $I$ incident plane waves. Then the $k$-th frequency component of an output of the $n$-th microphone is expressed using Eq. (11') as follows:

$$
X_{n}(k)=\sum_{i=1}^{I} A_{i}(k) z_{i}^{n},
$$

where

$$
\begin{aligned}
z_{i} & =\exp \left(-j 2 \pi \frac{k}{T} d \cos \theta_{i} / c\right), \quad i=1,2, \cdots, I \\
& =\exp \left(j \phi_{i} d\right) .
\end{aligned}
$$

When a complex sequence expressed by Eq. (24) is given for $-\infty<n<+\infty$, the true auto-correlation function $c(l)$ is expressed as follows:

$$
\begin{aligned}
c(l)= & \lim _{N \rightarrow \infty} \frac{1}{2 N-1} \sum_{n=1-N}^{N-1} X_{n}(k) X_{n-l}^{*}(k) \\
= & \lim _{N \rightarrow \infty} \frac{1}{2 N-1} \sum_{n=1-N}^{N-1} \cdot \sum_{i=1}^{I} A_{i}(k) z_{i}^{n} \\
& \cdot \sum_{m=1}^{I} A_{m}^{*}(k) z_{m}^{l-n} \\
= & \sum_{i=1}^{I}\left|A_{i}(k)\right|^{2} z_{i}^{l} .
\end{aligned}
$$

The sequence is practically given for a finite value $n$, where $0 \leqq n \leqq N-1$. Therefore, the auto-correlation function $v(l)$ is calculated assuming that $X_{n}(k)$ $=0$ for $n<0$ and $n \geqq N$ as follows:

$$
v(l)=\frac{1}{N-|l|} \sum_{n=0}^{N-1} X_{n}(k) X_{n-l}^{*}(k) .
$$

In Eq. (26), the true auto-correlation function $c(l)$ is the function of $2 I$ parameters: $\left|A_{i}(k)\right|^{2}$ and $z_{i}$, 
where $i=1,2, \cdots, I$. To estimate the $2 I$ parameters, it is assumed that true auto-correlation functions $c(l)$ are approximately equal to the calculated autocorrelation functions $v(l)$ as follows:

$$
c(l) \fallingdotseq v(l), \quad l=0,1,2, \cdots, 2 I-1 .
$$

From Eqs. (26) and (28), the following equations are obtained:

$$
\begin{aligned}
\sum_{i=1}^{I}\left|A_{i}(k)\right|^{2} z_{i}^{l} \fallingdotseq v(l), \\
\quad l=0,1,2, \cdots, 2 I-1 .
\end{aligned}
$$

Here, polynomial $B_{I}(z)$ of the order $I$ which falls 0 at $z=z_{i}$ is introduced as follows:

$$
\left.\begin{array}{l}
B_{I}(z)=\sum_{q=0}^{I} b_{q} z^{q}=\prod_{i=1}^{I}\left(z-z_{i}\right) \\
b_{I}=1
\end{array}\right\} .
$$

Multiplying both sides of Eq. (29) by $B_{I}\left(z_{i}\right)$, the following equations are obtained:

$$
\begin{aligned}
\sum_{i=1}^{I}\left|A_{i}(k)\right|^{2} B_{I}\left(z_{i}\right) z_{i}^{l} & =\sum_{i=1}^{I}\left|A_{i}(k)\right|^{2} \sum_{q=0}^{I} b_{q} z_{i}^{q+l} \\
& =\sum_{q=0}^{I} b_{q} \sum_{i=1}^{I}\left|A_{i}(k)\right|^{2} z_{i}^{q+l} \\
& \fallingdotseq \sum_{q=0}^{I} b_{q} v(q+l) .
\end{aligned}
$$

The following equation is obtained from Eq. (31) because $B_{I}\left(z_{i}\right)=0$ and that $b_{I}=1$.

$$
\begin{array}{r}
\sum_{q=0}^{I-1} b_{q} v(q+l) \doteqdot-v(I+l), \\
l=0,1,2, \cdots, 2 I-1 .
\end{array}
$$

In Eq. (32), $b_{q}$ 's, where $q=0,1, \cdots, I-1$, are unknown; the number of unknown parameters is $I$. However, there are $2 I$ equations in (32). Therefore, $b_{q}$ 's which satisfy all equations in (32) can not always be obtained. Thus, $b_{q}$ 's are calculated by solving the first $I$ equations;

$$
\begin{gathered}
{\left[\begin{array}{ccccc}
v(0) & v(1) & v(2) & \cdots & v(I-1) \\
v(1) & v(2) & v(3) & \cdots & v(I) \\
\vdots & \vdots & \vdots & \cdots & \vdots \\
\vdots & \vdots & \vdots & \cdots & \vdots \\
v(I-1) & \cdots \cdots \cdots \cdots \cdots \cdots & v(2 I-2)
\end{array}\right]\left[\begin{array}{c}
b_{0} \\
b_{1} \\
\vdots \\
\vdots \\
b_{I-1}
\end{array}\right]} \\
\vdots-\left[\begin{array}{c}
v(I) \\
v(I+1) \\
\vdots \\
v(2 I-1)
\end{array}\right],
\end{gathered}
$$

$z_{i}$ 's are calculated by solving the following polynomial, whose coefficients $b_{q}$ 's are obtained approximately by Eq. (33).

$$
B_{I}(z)=z^{I}+b_{I-1} z^{I-1}+\cdots+b_{1} z+b_{0}=0
$$

Since $b_{q}$ 's are the approximate value, $z_{i}$ 's obtained by Eq. (34) do not always satisfy Eq. (25); $z_{i}$ 's are a little different from the true value. Furthermore, if the assumed number $I$ is not equal to the true number $P$ of incident plane waves, $z i$ 's may be very different from the true value; in the case $I<P, z_{i}$ 's are not estimated correctly; in the case $I \geqq P$, empirically $P$ out of $I \quad z_{i}$ 's are estimated almost correctly and the rest are false. Then, $z_{i}$ 's can be expressed as follows:

$$
\begin{aligned}
z_{i} & =\alpha_{i} \exp \left(-j 2 \pi \frac{k}{T} d \cos \theta_{i} / c\right) \\
& =\alpha_{i} \exp \left(j \phi_{i} d\right), \quad i=1,2, \cdots, I,
\end{aligned}
$$

where $\alpha_{i}$ 's $>0$, and $\theta_{i}$ and $\phi_{i}$ are not always equal to the true ones. Since the spatial sequence on the array is composed of non-decaying complex sinusoidal waves, $\alpha_{i}$ 's should be equal to 1 . Thus, the power spectrum $\left|A_{i}(k)\right|^{2}$ and the direction $\theta_{i}$ of the $i$-th incident plane wave are estimated by putting $\alpha_{i}=1$ in Eq. (35),

$$
\begin{aligned}
z_{i}^{\prime} & =\exp \left(-j 2 \pi \frac{k}{T} d \cos \theta_{i} / c\right) \\
& =\exp \left(j \phi_{i} d\right), \quad i=1,2, \cdots, I .
\end{aligned}
$$

Because of the same reason as in the case of $z_{i}$, the PCR $\phi_{i}=(2 \pi k / T c) \cos \theta_{i}$ does not always satisfy Eq. (10). Thus, $z_{i}^{\prime}$ 's are replaced or eliminated according to the value of $\phi_{i}$. That is, $z_{i}$ 's should be eliminated when $\phi_{i}$ 's do not satisfy Eq. (10). However, even in the case where $\phi_{i}$ should theoretically satisfy Eq. (10), $\phi_{i}$ sometimes takes a value which does not satisfy Eq. (10) because $\phi_{i}$ is computed using approximate equation. Therefore, such $\phi_{i}$ should not be discarded and the following equation is established using a small positive value $\Delta \phi$ :

$$
z_{i}^{\prime}= \begin{cases}\exp \left(j \phi_{i} d\right), & \left|\phi_{i}\right|<\frac{2 \pi k}{T c} \\ \exp \left(j 2 \pi \frac{k d}{T c}\right), & \frac{2 \pi k}{T c} \leqq \phi_{i}<\frac{2 \pi k}{T c}+\Delta \phi \\ \exp \left(-j 2 \pi \frac{k d}{T c}\right), & -\left(\frac{2 \pi k}{T c}+\Delta \phi\right)<\phi_{i} \leqq-\frac{2 \pi k}{T c} \\ \text { eliminated, otherwise }\end{cases}
$$


In the computer simulation explained in section $3, \Delta \phi$ is set to be $\pi k /(5 T c)$. The estimated number $I^{\prime}$ of incident plane waves is set to the number of $z_{i}^{\prime}$ 's which are not eliminated. Since the true number $\boldsymbol{P}$ of incident plane waves is unknown, direction and spectrum are estimated for various $I$ which is assumed to be the number of incident plane waves. In the case $I<P$, the error in the estimation is large; in the case $I \geqq P$ the error becomes small by using estimated number $I^{\prime}$ instead of the assumed number $I$ because $I^{\prime}$ may be equal to $P$.

The power spectrum $\left|A_{i}(k)\right|^{2}$ of the $i$-th incident plane wave, where $i=1,2, \cdots, I^{\prime}$, is calculated from Eq. (29) using $z_{i}^{\prime}$, i.e., by solving the following equations:

$$
\begin{gathered}
{\left[\begin{array}{cccc}
1 & 1 & \cdots \cdots & 1 \\
z^{\prime}{ }_{1} & z^{\prime}{ }_{2} & \cdots \cdots & z_{I^{\prime}}^{\prime} \\
\vdots & \vdots & \cdots \cdots & \vdots \\
\vdots & \vdots & \cdots \cdots & \vdots \\
z^{\prime I^{\prime}-1} & z_{2}^{\prime I^{\prime}-1} & \cdots \cdots & z^{\prime I^{\prime}-1}
\end{array}\right]\left[\begin{array}{c}
\left|A_{1}(k)\right|^{2} \\
\left|A_{2}(k)\right|^{2} \\
\vdots \\
\vdots \\
\left|A_{I^{\prime}}(k)\right|^{2}
\end{array}\right]} \\
\vdots\left[\begin{array}{c}
v(0) \\
v(1) \\
\vdots \\
\vdots \\
v\left(I^{\prime}-1\right)
\end{array}\right] .
\end{gathered}
$$

The direction $\theta_{i}$ of the $i$-th incident plane wave is calculated from Eq. (25) also using $z_{i}^{\prime}$.

Above processing is executed for $I=1,2, \cdots, M$, where $M$ is the assumed maximum number of incident plane waves. The power spectra $\left|A_{i}(k)\right|^{2}$ and the direction $\theta_{i}$ of the $i$-th incident plane wave are estimated using the number $I^{\prime}$ and $z_{i}^{\prime}$ obtained when the following prediction error $P_{I, I^{\prime}}$ reaches the smallest value.

$$
\boldsymbol{P}_{I, I^{\prime}}=\left.\sum_{l=0}^{N-1}\left|\sum_{i=1}^{I^{\prime}}\right| A_{i}(k)\right|^{2} \cdot z_{i}^{\prime \prime}-\left.v(l)\right|^{2} .
$$

\section{COMPUTER SIMULATION}

In this section, the three methods described in section 2 are compared with one another by computer simulation. In the simulation, there are three sound sources as shown in Fig. 4. Each sound source radiates white noise having no correlation between one another. The conditions of simulation are that the sampling frequency is 100 $\mathrm{kHz}$, the length of Hanning window $0.02048 \mathrm{~s}$, the number of microphones 101 , the distance be-

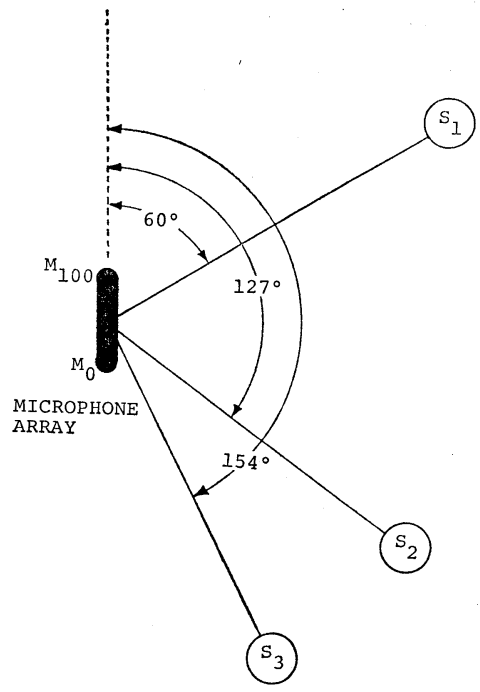

Fig. 4 Relation between a microphone array and 3 sound sources for computer simulations.

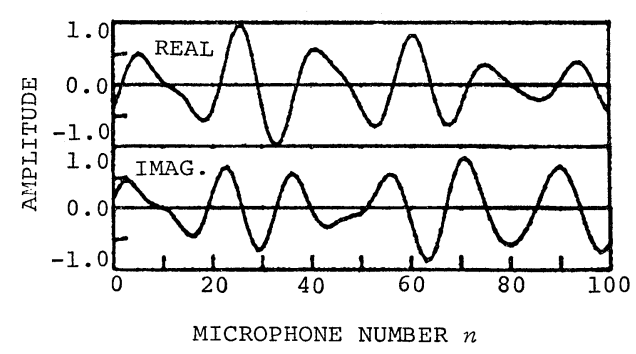

Fig. 5 A spatial sequence obtained by arranging $977 \mathrm{~Hz}$ components according to the order of microphone number.

tween adjacent two microphones $3.4 \mathrm{~cm}$ and the length of the array $3.4 \mathrm{~m}$.

Figure 5 shows the sequence obtained by arranging $977 \mathrm{~Hz}$ components according to the order of the microphone number. It is clear that the sequence is composed of a number of complex sinusoidal waves. The power spectrum and direction of every incident sound can be estimated by evaluating amplitude and PCR of the corresponding complex sinusoidal wave.

Figures 6(a), (b) and (c) show the results analyzed by the three methods: DFT, MEM and CCSM, respectively. All of the results show peaks in the 

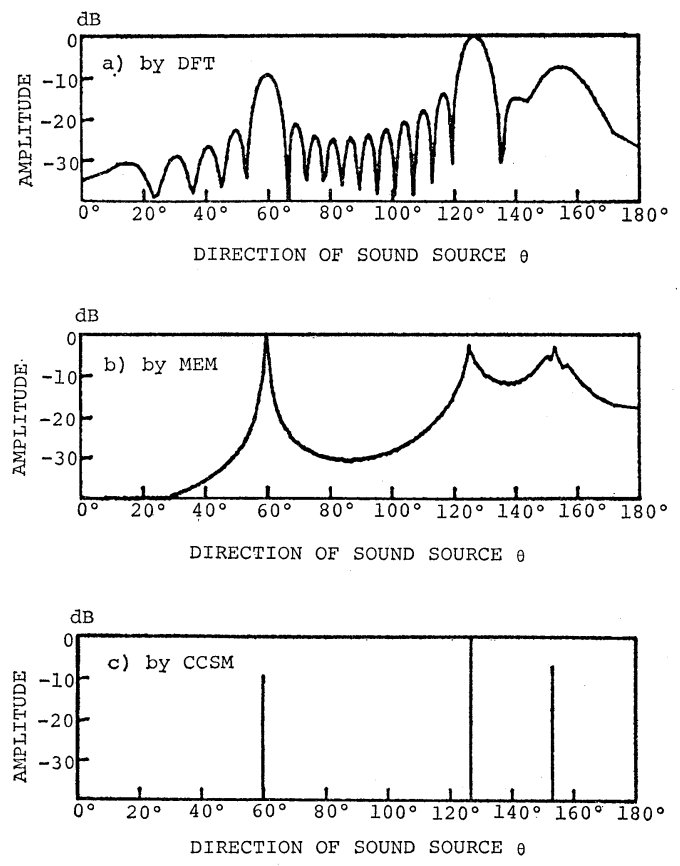

Fig. 6 Results of computer simulation for $977 \mathrm{~Hz}$ component.

(a) DFT, (b) MEM, (c) CCSM.

directions from which the three incident plane waves come. However, there are considerable differences among the results.

Figure 6(a) obtained by DFT shows many side lobes. The peaks due to the side lobes are often taken for the incident sounds, or the incident sound can not be detected if the peak due to the incident sound is smaller than the peaks due to the side lobes.

There are no side lobes in Fig. 6(b) obtained by MEM. This figure shows, however, a relatively broad main lobe which is apt to mask the peak due to the smaller incident sound especially when the incident direction of the sound is close to that of the larger one.

Figure 6(c) obtained by CCSM shows only three lines indicating the directions of incident sound sources; the problems involved in the other methods are completely dissolved.

Figure 7 shows the sequence which is obtained by arranging $146 \mathrm{~Hz}$ components according to the order of the microphone number. Figures $8(\mathrm{a})$, (b) and (c) show the results analyzed by the three

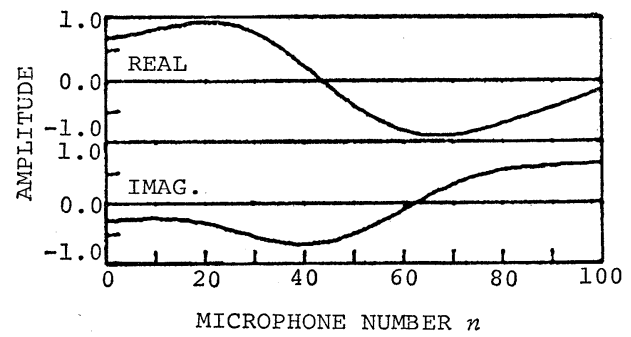

Fig. 7 A spatial sequence obtained by arranging $146 \mathrm{~Hz}$ components according to the order of microphone number.
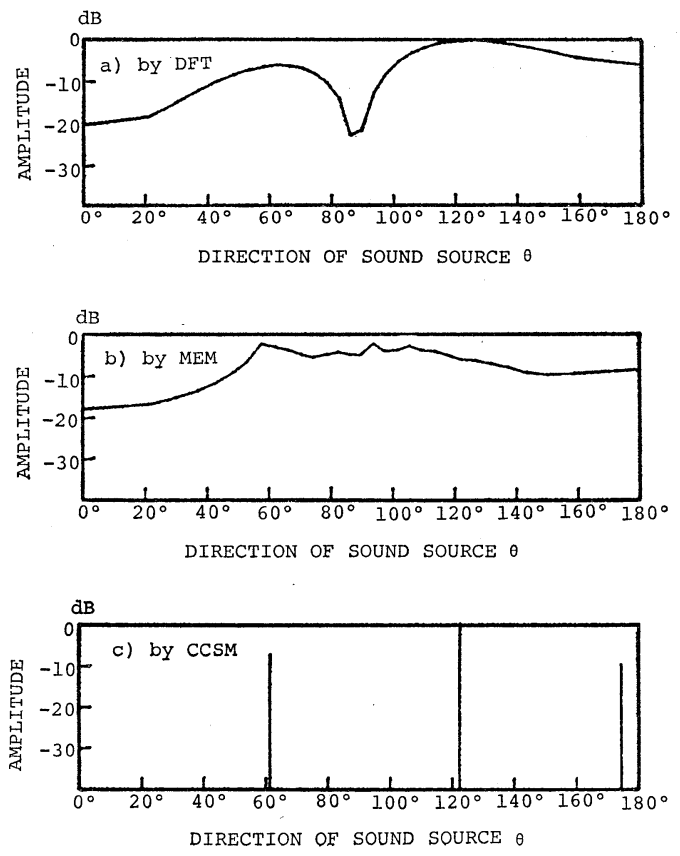

Fig. 8 Results of computer simulation for $146 \mathrm{~Hz}$ component.

(a) DFT, (b) MEM, (c) CCSM.

methods. In Fig. 8(a) obtained by DFT, the sound source $S_{1}$ in $154^{\circ}$ direction can not be separated from that in $127^{\circ}$ direction because the length of the array is of the same order as the wave length of sound. In Fig. 8(b) obtained by MEM, there are no explicit peaks, while Fig. 8(c) which is obtained by CCSM shows three lines showing the directions of incident sound sources, although the estimated directions are somewhat different from the true directions. 


\section{CONCLUSION}

Together with its application to the array signal processing, composite complex sinusoidal modeling (CCSM) is proposed as a new method for analyzing a signal composed of a number of complex sinusoidal waves.

Output of every microphone in a linear array is stored in a computer. Every stored sequence is cut out by a proper time window and separated into different frequency components by Fourier transform. After selecting the same frequency components from the transformed data of the outputs of all microphones, a new sequence is generated by arranging the components according to the order of the microphone number. When there are a number of plane waves, a new sequence is composed of the sum of the same number of spatial complex sinusoidal waves as that of the incident plane waves. Direction and power of the frequency component of each plane wave is estimated by evaluating amplitude and PCR of the corresponding complex sinusoidal waves. Therefore, an arbitrary method for the spectral analysis can be used for estimating power and direction of an incident plane wave. Thus, discrete Fourier transform (DFT), maximum entropy method (MEM) and composite complex sinusoidal modeling (CCSM) are compared with one another.

Accuracy obtained by DFT is not so good unless the length of the array is sufficiently longer than the wave length of the sound, because the directivity pattern has many side lobes with a rather broad main lobe. That is, the main lobe is very broad and apt to mask the smaller peak due to the incident sounds from other directions when the length of the array is of the same order as the wave length of the sound. The directivity pattern obtained by MEM has no side lobes but is not so sharp when the length of array is of the same order as the wave length. Furthermore, accurate estimation can not be expected because application of MEM means that the spatial sequence composed of complex sinusoidal waves is approximated by all pole modeling. Meanwhile, CCSM is the best because it is based on the model in which a signal is composed of complex sinusoidal waves. The directivity pattern obtained by CCSM gives only a single line indicating the direction of an incident sound. Computer simulations show that accuracy obtained by CCSM is much better than that obtained either by DFT or by MEM especially when the length of the array is of the same order as the wave length.

\section{REFERENCES}

1) S. Sagayama and F. Itakura, "Composite sinusoidal modeling applied to spectral analysis of speech," I.E.C.E. J64-A, 105-112 (1981) (in Japanese).

2) M. Abe and K. Kido, "An application of composite complex sinusoid analysis method to the detection of the direction of incident sound," Tech. Rep. I.E.C.E. EA80-80, 15-22 (1981) (in Japanese).

3) J. P. Burg, "Maximum entropy spectral analysis," the 37th Annual International Meeting, Soc. Explor. Geophys., Oklahoma City, Oklahoma (1967).

4) H. P. Bucker, "High-resolution cross-sensor beamforming for a uniform line array," J. Acoust. Soc. Am. 63, 420-424 (1978).

5) D. P. Skinner, S. M. Hedlicka, and A. D. Matthews, "Maximum entropy array processing," J. Acoust. Soc. Am. 66, 488-493 (1979).

6) J. D. Markel and A. H. Gray, Jr., "On autocorrelation equations as applied to speech analysis," IEEE Trans. AU-21, 69-79 (1973). 